\title{
SCIDOC
}

\author{
International Journal of Dentistry and Oral Science (IJDOS) \\ ISSN: 2377-8075
}

\section{Stress, Anxiety and Depression Among Dental Students in Times of Covid-19 Lockdown}

Aya Abu Kwaik, Raghad Saleh, Mayar Danadneh, Elham Kateeb*

Oral Health Research and Promotion Unit, Faculty of Dentistry, Al-Quds University, Jerusalem, State of Palestine.

\section{Abstract}

Introduction: Dental students in their clinical training, face a higher risk of contracting the disease as well as experiencing adverse psychological outcomes. Therefore, this study came to evaluate the impact of COVID-19 and the lockdown on the mental health of dental students during the current pandemic.

Methods: This is a cross-sectional study conducted among dental students during the first months of the COVID-19 pandemic to assess the levels of depression, anxiety, and stress using the standardized DASS-21 scale. In addition demographic, socio-economic, academic performance, source of information about COVID-19, and perception of on-line education difficulties were collected.

Results: A total of 436 students completed the online survey (55.18\% response rate). In the current sample, $48 \%(\mathrm{n}=209)$, $76 \%(n=331), 70 \%(n=305)$ showed different levels of stress, anxiety and depression. Students who stated that their family income was impacted by COVID-19 showed signs of depression $X 2=7.3, p=.007$ and anxiety, $X 2=6.1, p=.013$. Dental students who faced difficulties in switching to E-learning, reported different levels of depression, $\mathrm{X} 2=14, \mathrm{p}<.001$, anxiety, $\mathrm{X} 2=9, \mathrm{p}=.003$ and stress, $\mathrm{X} 2=24, \mathrm{p}<.001$.

Conclusions: In preparation for the next academic year, strategies to support the mental health of dental students in their clinical training and E-learning process should be in place.

Keywords: Depression; Anxiety; Stress; COVID-19; Dental Students.

\section{Introduction}

On March 11th, 2020, the World Health Organization (WHO) declared the COVID-19, a pneumonia-like disease with severe symptoms and high transmissibility (Wang et al., 2020), as an international pandemic ((WHO), 2020). COVID-19 is caused by a 2019 novel coronavirus, SARS-COV-2 virus, that was first identified in Wuhan, China in December 2019, and then spread out to the rest of the world (Ryu S et al., 2020). At the time of writing, the worldwide cases of COVID-19 are steadily increasing across all continents with a cumulative total of individuals presented with confirmed COVID-19 is 22,256,220 people, and 782,456 deaths reported to the World Health Organization((WHO), 2020). In Palestine, a rapid surge in the number of COVID-19 cases was observed during the first period of the pandemic. In the time of data collection for this study and according to the Palestinian Ministry of Health official site, there were 115 confirmed cases in the West Bank, Gaza and East Jerusalem, and one case of death for a sixty-year-old woman in Jerusalem suburbs. However, now, the number of confirmed cases according to the Ministry of Health official website, has reached 21,554 people and 119 deaths ((MOH), 2020).

Once the first 5 cases were identified in Bethlehem area, local authorities in Palestine called for partial closures on March 5th followed by a total lockdown on March 22nd including most aspects of life. Lockdown at different degrees was the most common practice in many countries as the only available way to contain the outbreak in its beginning. According to the International Journal of Information and Systems Sciences; quarantine and social distancing have been the most effective techniques to contain and reduce outbreaks of contagious diseases as long as the appropriate treatment or vaccine is still not known (Yan et al., 2007).

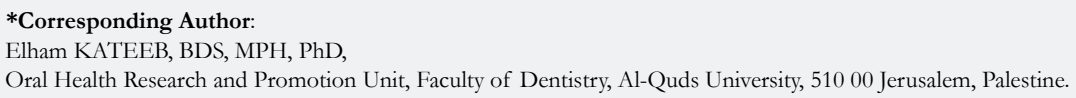

Copyright: Elham KATEEB ${ }^{\circ}$ 2021. This is an open-access article distributed under the terms of the Creative Commons Attribution License, which permits unrestricted use, distribution and reproduction in any medium, provided the original author and source are credited. 
National lockdown measures started in Palestine on March 5th and extended to May 26th, with some relief measures occurring afterward. The national lockdown included the closure of all public facilities; worship places, restaurants, recreation facilities, schools, universities and governmental institutions. It also imposed cancelation or postponement of all types of festivities and closure of the borders As a consequence, the closures impacted various industry sectors; including the pharmaceutical, tourism, information and electronics industries ((MOH,2020).

Prior research revealed a profound and broad range of psychosocial impacts during outbreaks of infection on people at the individual, community, and international levels. People are likely to experience fear of falling sick or dying on an individual level, feelings of helplessness and stigma(Hiremath et al., 2020) The disease itself multiplied by forced quarantine to combat COVID-19 applied by nation lockdowns can produce acute panic, anxiety, obsessive behaviors, hoarding, paranoia, and depression, and post-traumatic stress disorder (PTSD) in the long run (Dubey et al., 2020). These have been fueled by an "infodemic" spread via different platforms of social media.

In addition to all of the above, healthcare workers including dentists and dental students in their clinical training, face a higher risk of contracting the disease as well as experiencing adverse psychological outcomes in form of burnout, anxiety, fear of transmitting infection, feeling of incompatibility, depression, increased substance-dependence, and PTSD (Dubey et al., 2020).

On March 5th and as part of the first closure, Al-Quds University (AQU), one of the biggest universities in the middle of the West Bank area, among other universities in Palestine stopped physical attendance and switched totally online for theoretical courses. As a response and for the sake of students' safety, the dental school discontinued all clinical and preclinical training. Uncertainty of what would happen next in their clinical training, their exams and their e-learning, left homebound Palestinian dental students helpless and confused about their educational outcomes. Moreover, the suspension of most professions and the negative impact of lockdown on the economic status led to a general atmosphere of anxiety and depression about financial stability and to a huge disruption in the way people used to live (Godinic et al., 2020). Therefore, this study came to evaluate the impact of COVID-19 and the lockdown on the mental health of dental students at AlQuds University during the first months of the pandemic. Specifically, it assessed the level of Depression, Anxiety, and Stress levels among dental students during pandemic using the standardized DASS-21 scale.

\section{Methods}

Data was collected cross-sectionally in the period between March 28th, 2020 and April 10th, 2020, three weeks after the university closure and the national lockdown implementation on March 5th. Dental students in their different years of study from Al-Quds University (AQU) received an online survey posted on their official Facebook groups and sent to them via the messenger app. Out of the 870 students registered at AQU Dental Faculty, 790 students were enrolled in the school official Facebook groups and had access to the survey link. Sample size calculation was carried out using Raosoft online calculator (Raosoft, 2015) estimated 356 as a minimum sample needed to achieve $95 \%$ confidence level and $4 \%$ margin of error.

The Arabic version of a 21-item version of the Depression Anxiety Stress Scales (DASS-21) (Antony et al., 1998) was used in this study to measure levels of depression, anxiety, and stress among dental students (L. S. Lovibond PF et al., 1995). DASS-21 scale has shown good reliability and validity in both English and Arabic versions (Ali et al., 2017). To check the reliability of the scales in the current study, we calculated Cronbach's alpha for the entire scale and the three subscales. In our study, Cronbach's alpha was 0.884 , for the entire scale, and $0.84,0.8,0.88$ for the depression, anxiety, and stress scales respectively, all of which indicate very good internal consistency and therefore very good reliability.

Scores for the he DASS-21 3 subscales were obtained from summing up the scores of the 7 statements related to each construct (Stress, Anxiety and Depression). DASS-21 is the short form of the DASS-42, thus, subscales' scores were multiplied by 2 to represent the total for the full subscale DASS-42. Higher scores indicate higher levels of the condition and ranged between 0 and 42. The scores for each subscale are then categorized to indicate the level of severity of each condition ranging from normal, mild, moderate, severe and extremely severe according to cut points suggested by Lovibond, S.H. \& Lovibond, P.F. (Lovibond, S.H. \& Lovibond, P.F. (1995)). To calculate the prevalence of the condition, the 5 categories were dichotomized into ( presence and absence of the condition) combining the four severity levels (mild, moderate, severe and extremely severe) to one category.

In addition to the DSS-21 scales, demographic, socio-economic , academic performance, source of information about COVID-19, and perception of on-line education difficulties were collected.

Statistical analysis was conducted using the Statistical Package for the Social Sciences (SPSS Inc. Chicago, IL. 2020) version 23.0. Frequency and percentages were generated for all study variables. Bivariable associations were investigated among study variables using Pearson Chi-square and t- test. Significance level was set to 0.05 .

The study was conducted in accordance with the Declaration of Helsinki and reported according to the Checklist for Reporting Results of Internet E-Surveys (CHERRIES) ((WMA),2013), (Eysenbach G. 2004) All aspects of the study were approved by the Al-Quds University Ethics Committee.

\section{Results}

A total of 436 students completed the online survey with a response rate of $55.18 \%$. Eighty-one present $(n=354)$ of our sample were females. Sample demographic and socioeconomic characteristics are presented in Table 1 .

Fifty-eight percent $(n=255)$ of our sample reported having "very good" or "excellent" academic performance and $45.5 \%(n=181)$ reported having "good" or "fair" academic performance. Students in this sample spent on average $4 \pm 2.2$ hours on E-learning (online education) and $62.2 \%(n=271)$ reported that they face difficulties in this mode of learning. Eighty-five percent of participants $(n=369)$ were interested in news updates about COVID-19 
news and $42.9 \%(n=192)$ used social media as their main source of information. Sources of COVID-19 news used by the students are found in Figure 1.

Among our sample and by the time of data collection, 6 participants had family members or friends who were infected by the COVID-19 $(1.4 \%)$ and 205 students had family members in quarantine (47\%). When we asked if the lockdown had a negative impact on the economic status of the family, $75.7 \%(n=330)$ responded "Yes".

Students in this sample scored on average on a scale of (0-42), $15.5 \pm 10$ for Stress, $15.6 \pm 10$ for Anxiety, and 15.6 \pm 10 for De- pression. Forty-eight percent $(n=209)$ showed signs of stress at different levels, $76 \%(\mathrm{n}=331)$ showed different levels of anxiety and $70 \%(n=305)$ showed different levels of depression. Levels of severity according to the selected cut points for the three subscales are found in Table 2.

When we tried to understand factors related to the presence of any level of the three conditions, we found, in general, students who rated their academic performance better, had less stress, $\mathrm{X} 2=11.5$, $\mathrm{p}=.003$, less anxiety $\mathrm{X} 2=7.6, \mathrm{p}=.023$, and less depression, $\mathrm{X} 2=7.1$, $\mathrm{p}=.029$. In this sample, students who stated that their family income was impacted by COVID-19 showed different levels of Depression $\mathrm{X} 2=7.3, \mathrm{p}=.007$ and Anxiety, X2=6.1, $\mathrm{p}=.013$. In

Table 1. Sample demographic and socioeconomic characteristics.

\begin{tabular}{|c|c|c|}
\hline Variables & Categories & Number (\%) \\
\hline \multirow{3}{*}{ Years of Study } & first Year & $75(17.2)$ \\
& Second Years & $73(16.7)$ \\
& Third Years & $88(20.2)$ \\
& Fourth Years & $101(23.2)$ \\
& Fifth Years & $99(22.7)$ \\
\hline Gender & Male & $82(18.8)$ \\
& Female & $354(81.2)$ \\
\hline Type of Residence Place & City & $234(53.7)$ \\
& Village/Town & $193(44.3)$ \\
& Camp & $9(2.1)$ \\
\hline & Less than 294\$ & $6(1.4)$ \\
& $294-587 \$$ & $35(8.0)$ \\
& $587-880 \$$ & $60(13.8)$ \\
Average of Monthly Income/Family & $880-1175 \$$ & $81(18.6)$ \\
& $1175-1470 \$$ & $97(22.2))$ \\
& $1470 \$$ and more & $157(36.0)$ \\
\hline University tuition financing & Family funds & $402(92.2)$ \\
& Personal & $5(1.1)$ \\
& Scholarship & $16(3.7)$ \\
& Other & $13(3.0)$ \\
\hline
\end{tabular}

Table 2. Levels of the three conditions severity according to the selected cut points suggested by Lovibond, S.H. $\&$ Lovibond, P.F.

\begin{tabular}{|c|c|c|c|c|c|}
\hline & Normal & Mild & Moderate & Severe & Extremely Severe \\
\hline Depression & $(131) 30 \%$ & $(67) 15.4 \%$ & $(106) 24 \%$ & $(69) 15.8 \%$ & $(63) 14.4 \%$ \\
\hline Anxiety & $(105) 24 \%$ & $(26) 6 \%$ & $(91) 20.9 \%$ & $(55) 12.6 \%$ & $(159) 36.5 \%$ \\
\hline Stress & $(227) 52.1 \%$ & $(30) 15.1 \%$ & $(60) 13.8 \%$ & $(83) 19 \%$ & $(0) 0 \%$ \\
\hline
\end{tabular}

Figure 1. Sources of Information used by Dental Students to update their Knowledge about COVID-19.

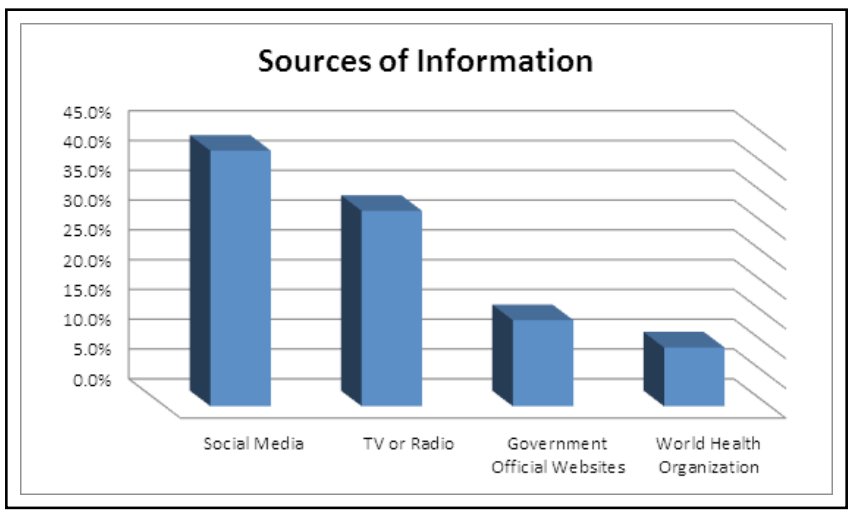


addition, dental students who faced difficulties in switching to e-learning, had different levels of Depression, X2 $=14, \mathrm{p}<.001$, Anxiety, X2=9, p=.003 and Stress, X2=24, $<<.001$. Students who followed updated news about COVID-19 had less anxiety, $\mathrm{t}=$ 2.58, $\mathrm{p}=.031$ and less depression $\mathrm{t}=-2.6, \mathrm{p}=.031$; however this did not affect their stress levels, $t=-.37, \mathrm{p}=.71$.

\section{Discussion}

This study assessed the psychological distress (Stress, Anxiety, Depression) among a convenience sample of Palestinian dental students after few weeks of closing universities, switching to e-learning and shutting down all aspects of life in response to the COVID-19 pandemic. Results of this study give insights of sources of psychological distress among dental students during the pandemic and enable dental schools to plan interventions to support students' mental health in this difficult time. Dental students bear an extra burden in the lockdown, which is the complete suspension of clinical training that is considered a core curriculum requirement to pass any course or to proceed to the year after. Online education can't completely address the challenges and the requirements of dental education which put dental students under additional stress. Majority of students in this sample showed different levels of depression and anxiety and to a lesser extent, stress. Half of the dental students in this sample had "Sever" or "Extremely Severe" anxiety and one third had "Severe" or " Extremely Severe" depression. According to the American Psychiatric Association (www.psychiatry.org/), stress and anxiety are both emotional responses, but stress is typically caused by an external trigger and for a short time compared to anxiety, which is defined by persistent, excessive worries that don't go away even in the absence of a stressor. Depression is a single illness that is defined by having a low mood and/or a loss of interest or enjoyment in most activities, for two weeks or longer.

To the best of our knowledge, no studies were conducted to measure stress, anxiety and depression among dental students in the current pandemic; however, a study that was conducted among students and health workers in a university setting in Spain during COVID-19 pandemic using DASS-21 demonstrated less severity of these conditions (Odrizola et al., 2020). Thirty - five percent, $48.10 \%$ and $40.32 \%$ of the survey respondents in the previous study exhibited signs of psychological symptoms according to anxiety, depression and stress scores, respectively. In another study in a university setting in Istanbul, Turkey among health workers and using DASS-21 during the current pandemic, of all 442 participants, $64.7 \%$ had symptoms of depression, $51.6 \%$ anxiety, and $41.2 \%$ stress (Elbay et al., 2020). In both studies the prevalence of the three conditions were less than the current study.

Major two stressors in the COVID-19 lockdown times were evident in this study, financial impact of the lockdown, and switching to online learning. Financial impact was very profound on the fragile Palestinian economy and this greatly impacted the prevalence of the three conditions in the current sample. The Palestine Economic Policy Research Institute (MAS) demonstrated in a recent report that the Palestinian economy has already entered a recession and there is already a major decline in all economic indicators (Habib et al., 2020).
Switching to e-learning or online education was necessary to comply with the strict measures of social distancing that aim to minimize disease transmission. Online education cannot by any means cover dental clinical training, therefore students' psychological distress about how they would resume safely their clinical training is for sure is a major stressor in this pandemic. Dental clinical training in student dental clinics, in particular, is considered at very high risk of disease transmission because of the close proximity between the care provider and the patient and the aerosols generating nature of the most dental treatment procedures (Vergara-Buenaventura et al.,2020).

This study is cross-sectional and the nature of data collection method cannot indicate directionality of the cause and the outcome. In addition, this study was conducted 3 weeks after the lockdown was imposed, thus it assesses the psychological distress related to the sudden new changes happened to students' lives, but at the same time, it couldn't assess the accumulative effect of the lockdown and the suspension of all aspects life for longer periods. Therefore, follow up studies are needed to accurately investigate the impact of COVID-19 and the imposed lockdown on dental students.

In a few weeks, the new academic year is starting all over the world, and no treatment or vaccine are identified yet. Social distancing, mask-wearing, hand hygiene and avoiding mass gathering are still the only measures available to contain the disease (Yan et al.,2007). In an atmosphere of uncertainty, online teaching is still in effect in most global institutions for the fall semester of 2020 (Sahu, P. 2020) and clinical training is returning back with very strict measures and a threat to be suspended in any minute if a surge in cases occur. Thus, authors of this study expect that psychological distress related to the current pandemic is not going to decrease. Early strategies are needed for the prevention and treatment of the psychological effects created by COVID-19 pandemic. Educational institutions and in particular, dental schools need to design plans and programs to help mitigate stress, anxiety and depression. Peking University in China prepared a mental health handbook to provide instructions on how to cope with stress and other psychological problems produced by the COVID-19 pandemic and implemented specialized telephone hotlines to provide psychological counseling. Another important stressor is the unfiltered information broadcast by the news media and social media (Bendau at el.,2020). Among the current sample, social media was the main source of information about the current pandemic. Circulating alarming videos on COVID-19 freely, accessible to virtually everyone, especially to young people including students, could contribute negatively to their mental health.

\section{Conclusion}

This study suggests two strategies to minimize the psychological distress among dental students while they are preparing themselves now to start the new academic year under the COVID-19 response measures. First, conduction education and awareness sessions among dental students about COVID-19 and its control measures in the dental clinical settings and on the credible sources of information they need to use to update their knowledge about COVID-19, second, increasing the effectiveness of online teaching by enhancing methods of delivering the content knowledge through the e-learning medium and by training the students on 
the skills needed to navigate the online teaching system to get the most of it.

\section{Acknowledgement}

The authors would like to thank each anonymously participating dental student of Al-Quds University (Palestine) who contributed to making this work possible.

\section{Data Availability Statement}

The data that supports the findings of this study are available from the corresponding author upon reasonable request.

\section{Author Contribution}

AA, RS and MD all participated in Conceptualization, Data curation, Methodology, Writing-original draft, EK is the supervisor of this project and did the formal analysis, contributed to the methodology, supervision, validation and writing \& editing.

\section{References}

[1]. Ali AM, Ahmed A, Sharaf A, Kawakami N, Abdeldayem SM, Green J. The Arabic Version of The Depression Anxiety Stress Scale-21: Cumulative scaling and discriminant-validation testing. Asian J Psychiatr. 2017 Dec;30:5658. Pubmed PMID: 28755630.

[2]. Bao Y, Sun Y, Meng S, Shi J, Lu L. 2019-nCoV epidemic: address mental health care to empower society. Lancet. 2020 Feb 22;395(10224):e37-e38. Pubmed PMID: 32043982.

[3]. Bendau A, Petzold MB, Pyrkosch L, Mascarell Maricic L, Betzler F, Rogoll $\mathrm{J}$, et al. Associations between COVID-19 related media consumption and symptoms of anxiety, depression and COVID-19 related fear in the general population in Germany. Eur Arch Psychiatry Clin Neurosci. 2020 Jul 20:1-9. Pubmed PMID: 32691135

[4]. DASS-21; Antony, Bieling, Cox, Enns, \& Swinson, 1998.

[5]. Dubey S, Biswas P, Ghosh R, Chatterjee S, Dubey MJ, Chatterjee S, Lahiri D, Lavie CJ. Psychosocial impact of COVID-19. Diabetes \& Metabolic Syndrome: Clinical Research \& Reviews. 2020 Sep 1;14(5):779-88.

[6]. Elbay RY, Kurtulmuş A, Arpacioğlu S, Karadere E. Depression, anxiety, stress levels of physicians and associated factors in Covid-19 pandemics. Psychiatry Res. 2020 Aug;290:113130. Pubmed PMID: 32497969.
[7]. Eysenbach G. Improving the quality of Web surveys: the Checklist for Reporting Results of Internet E-Surveys (CHERRIES). J Med Internet Res. 2004 Sep 29;6(3):e34. Pubmed PMID: 15471760.

[8]. Habib Hinn, Shams Hanieh. COVID-19 IN PALESTINE: ECONOMIC SLUMP, RISING VULNERABILITY AND LIMITED POLICY RESPONSE EUROMESCO SPOT-ON No16 - JUNE 2020

[9]. Lovibond PF, Lovibond $\mathrm{SH}$. The structure of negative emotional states: comparison of the Depression Anxiety Stress Scales (DASS) with the Beck Depression and Anxiety Inventories. Behav Res Ther. 1995 Mar;33(3):33543. Pubmed PMID: 7726811.

[10]. Lovibond SH, Lovibond PF. Manual for the depression anxiety stress scales. Psychology Foundation of Australia; 1996.

[11]. Odriozola-González P, Planchuelo-Gómez Á, Irurtia MJ, de Luis-García R. Psychological effects of the COVID-19 outbreak and lockdown among students and workers of a Spanish university. Psychiatry Res. 2020 Aug;290:113108. Pubmed PMID: 32450409.

[12]. Raosoft sample size calculator, accessed November 2015. Raosoft, Inc.

[13]. Ryu S, Chun BC; Korean Society of Epidemiology 2019-nCoV Task Force Team. An interim review of the epidemiological characteristics of 2019 novel coronavirus. Epidemiol Health. 2020;42:e2020006.Pubmed PMID: 32023775.

[14]. Sahu P. Closure of Universities Due to Coronavirus Disease 2019 (COVID-19): Impact on Education and Mental Health of Students and Academic Staff. Cureus. 2020 Apr 4;12(4):e7541. Pubmed PMID: 32377489.

[15]. Hiremath P, Suhas Kowshik CS, Manjunath M, Shettar M. COVID 19: Impact of lock-down on mental health and tips to overcome. Asian J Psychiatr. 2020 Jun;51:102088. Pubmed PMID: 32302964.

[16]. Vergara-Buenaventura A, Chavez-Tuñon M, Castro-Ruiz C. The Mental Health Consequences of Coronavirus Disease 2019 Pandemic in Dentistry. Disaster Med Public Health Prep. 2020 Jun 5:1-4. Pubmed PMID: 32498741.

[17]. Godinic D, Obrenovic B, Khudaykulov A. Effects of economic uncertainty on mental health in the COVID-19 pandemic context: social identity disturbance, job uncertainty and psychological well-being model. Int. J. Innov. Econ. Dev. 2020 Apr;6:61-74.

[18]. Health Minister of the State of Palestine (MOH), 2020.

[19]. Wang C, Horby PW, Hayden FG, Gao GF. A novel coronavirus outbreak of global health concern. Lancet. 2020 Feb 15;395(10223):470-473. Pubmed PMID: 31986257

[20]. World Health Organization (WHO), 2020

[21]. World Medical Association. World Medical Association Declaration of Helsinki: ethical principles for medical research involving human subjects. Jama. 2013 Nov 27;310(20):2191-4.

[22]. Yan X, Zou Y, Li J. Optimal quarantine and isolation strategies in epidemics control. World Journal of Modelling and Simulation. 2007;3(3):202-11. 